\title{
Detection of pathogenic bacteria: a genosensor approach
}

\begin{abstract}
Nowadays, detection of pathogenic bacteria is one of the major concerns in food, beverage drinking water and pharmaceutical industries. The traditional methods used for identification of bacteria involve detection of characteristic metabolites or cellular reproductive cycles, which is time-consuming, less sensitive, and misleading. From last few decades there has been a great advance in the strategies for detection of pathogenic bacteria by using micro- and nano-fabrication technologies. These technologies largely improved the sensitivity, specificity, limit of detection, and simultaneously reduced the complexity of handing, time and cost of detection. The paper will be focused on newly developed nano- and micro-scaled sensors, microfluidics, lab-on-achip, and array based technologies for the detection of different pathogenic bacteria.
\end{abstract}

Keywords: bacterial detection, bacterial sensor, genosensor, pathogenic bacteria
Volume 4 Issue 3 - 2017

\author{
Sandip Kumar Dash,' Ashok Kumar ${ }^{2}$ \\ 'Department of Zoology, Chikiti College, India \\ ${ }^{2}$ Department of Molecular Biosensors, AcSIR-Institute of \\ Genomics and Integrative Biology, India
}

Correspondence: Sandip Kumar Dash, Department of Zoology, Chikiti College, Chikiti, Ganjam, Odisha-76I0I0, India, Tel (+9I) 9438764504, Email dashsandipkumar@gmail.com

Received: January 31, 2017 | Published: April 10, 2017
Abbreviations: aMBs, allosteric molecular beacons; AuNPs, gold nanoparticles; CNTs, carbon nanotubes; CV, cyclic voltammetry; DPV, differential pulse voltammetry; dsDNA, double stranded dna; FRET, fluorescence resonance energy transfer; G-DNA, genomic DNA; GMO, genetically modified organisms; ITO, indium tin oxide; LOD, limit of detection; MB, molecular beacons; miRNA, microRNA; PCR, polymerase chain reaction; PNA, peptide nucleic acid; QCM, quartz crystal microbalance; QDs, quantum dots; ssDNA, single stranded dna; SPR, surface plasmon resonance

\section{Introduction}

Detection of pathogenic bacteria is one of the major concerns in food, beverage drinking water, and pharmaceutical industries. The conventional methods used for identification of bacteria involve detection of their characteristic metabolites or cellular reproductive cycles, which are complicated, time-consuming, less sensitive, and misleading. Nanomaterials like nanoparticles, nanotubes, and nanowires are nowadays used extensively to enhance the sensitivity of any detection. In addition to this, microfabrication technologies allowed for nanocomposite based high throughput screening of analytes. Biosensor is one of the simplest, economic, sensitive, and minute analytical devices which are becoming popular in the field of food analysis, bio-terrorism, environmental issues, and human health. ${ }^{1,2} \mathrm{~A}$ general biosensor includes a transducer-analytes composite, a processor, and a detector. It mainly reply on the intimate coupling of an analytes like whole cell, antibody, enzyme or nucleic acid to a physical transducer. The processor converts the biological signals into any of the amperometric, potentiometric, piezoelectric, thermal or optical signals. The signal is almost proportional to the concentration of analytes which is detected by the detector. ${ }^{3,4}$

Detection of pathogen specific DNA sequence is one of the most significant methods used in the clinical, environmental, horticulture or food analysis. This method is highly sensitive, specific, simple, and economic. This also allows label free detection of multiple samples in a very short duration of time. ${ }^{5}$ Analysis of specific gene sequences or changes in the sequence, offer a rapid, reliable, and accurate scope for the detection of pathogen in the environment, food or living organisms. In this paper, we have discussed the details of genosenors involving micro- and nano-technologies reported in last few years for detection of various pathogens.

\section{Optical fiber sensors}

Optical fiber Genosensors are based on a fiber optics. In these sensors ssDNA probe is immobilized at one end of the optic fiber and then hybridized with the fluorescent labeled complementary ssDNA from the pathogen. The flouroscence signal transduced through a series of internal reflections from one end to the other. ${ }^{6}$ The hybridization of complementary DNA is monitored by change in the fluorescence intensity.

\section{SPR sensors}

SPR sensor involves detection of the changes in optical properties due to change in the interfacial refractive index. Thiol-modified ssDNA probes can be immobilized onto Au through covalent interaction and then hybridized with the complementary ssDNA from pathogens to detect using SPR. ${ }^{7}$ Hsu et al., ${ }^{8}$ detected Mycobacterium tuberculosis by using SPR with a sensitivity of $-0.087 \mathrm{~dB} /(\mu \mathrm{g} / \mathrm{mL})$ and a highest sensitivity of $115 \mathrm{ng} / \mathrm{mL}$. Cheng et al.,9 used electrochemically deposited AuNPs on ITO-glass surface for immobilization of specific DNA probe. Then used the composite electrode to detect point mutation in apolipoprotein E gene through impedance spectroscopy and localized SPR for confirmation of Alzheimer's disease.

\section{MB sensors}

MBs are ssDNA with a stem-and-loop structure having a fluorophore at one end and a quencher on the other end of the stem. MB probes offer a scope of highly sensitive and specific detection of any analyte. Binding of a complementary ssDNA to the MB lead to the conformational changes in the $\mathrm{MB}$ and increase in the distance between quencher and fluorophore resulting in the emission of fluorescence. Weihong et al., ${ }^{10}$ developed a sensor based on MB immobilized glass matrix through avidin-streptavidin bridges. Operating cooperatively sensors are simple and economic methods for SNP analysis. A MB along with two ssDNA adaptors can be used for development of an operating cooperatively sensor for detection of SNPs in diagnostic, environment, and forensic applications. ${ }^{11}$ An 
electrochemical aMB based sensor was reported for detection of 10-9 - 10-6g/ml of E. coli. O157:H7 using DPV. ${ }^{12}$ Cai et al. ${ }^{13}$ fabricated a label-free aMB based sensor which can bind to streptavidin peroxidase polymer and generate catalytic currents in the presence of DNA or miRNA as targets. The LOD of the sensor was found to be 44 and 13.6amol for the DNA and let-7a miRNA, respectively.

\section{QD sensors}

The unique optical properties of QDs make them ideal probes for their use in genosensors for SNPs detection. ${ }^{14}$ The QDs can be used for conjugating specific ssDNA sequences to it and detect the complementary DNA. ${ }^{15}$ An FRET sensor can have very low LOD value. A QD-capture sequence conjugate was used to bind with the target DNA which on the other hand caused binding of a dye-reporter conjugate sequence in a sandwich fashion. The reporter dye and the target DNA formed a FRET donor-acceptor pair which was used for detection of DNA at 10-15 scale. ${ }^{16}$ Binding of very few numbers of copies of target DNA may produce strong FRET signal but no fluorescence is expected in case of non-specific DNA.

\section{Piezoelectric sensors}

Piezoelectric sensors are one of the most diversified applicable techniques used for detection of various biological analytes including DNA. Piezoelectric sensors have high natural frequency, excellent linearity, and high stability. QCMs consist of an oscillating crystal immobilized with ssDNA probe. Hybridization of the complementary DNA results in increase of mass and decrease in oscillating frequency. The change in oscillating frequency is proportional to the amount of hybridization and can be detected. A 19-mer ssDNA probe, complementary to stx2 gene of E. coli was immobilized onto a cantilever and then hybridized with the G-DNA from beef wash to detect E. coli. ${ }^{17}$ The LOD of the sensor was found to be about 700 CFUs.

\section{Colorimetric sensors}

Nowadays, colorimetric sensors are gaining their importance nucleotide conjugated nanoparticle is used for detection of target DNA from specific pathogens. A colorimetric sensor over other sensors because of their simplicity, sensitivity, and label-free detection. Oligo using AuNPs and graphene oxides was reported by $\mathrm{Li}$ et al., ${ }^{18}$ for the detection of DNA. The ssDNA-AuNPs conjugates can intercalate between graphene oxide layers but dsDNA-AuNPs or other folded RNA can't. By detecting the agglutination reaction, ssDNAs can be differentiated from that of dsDNAs and other folded RNAs.

\section{Electrochemical genosensors}

Electrochemical genosensors are one of the most simple, reliable, sensitive techniques in the field of both label-free and labeled detection. ${ }^{19}$ This usually involves monitoring either current or potential keeping the other fixed. The changes in these parameters are proportional to the density or charge of the analytes. Almost all the electrochemical genosensors involves a redox indicator like methylene blue or potassium ferricyanide. The interaction of these indicators with the immobilized probe or hybridized dsDNA is readout using $\mathrm{CV}, \mathrm{DPV}$ or electrochemical impedance. New redox indicators are offering greater discrimination between ssDNA and dsDNA. Some of the noncovalent attachments commonly used for immobilization of the ssDNA probe are epoxide-amine, epoxide-thiol, epoxide- aminooxyalkyl, aldehyde-amine, and semicarbazide-aldehyde whereas, the covalent linkages used are like carboxyl-amine, thioldisulfide, amine-aldehyde, aldehyde-oxyamine, biotin-streptavidin, gold-thiol, zirconylated-surface-phosphate and epoxide-amine. ${ }^{20,21}$ The fabrication of NPs like CNTs onto the transducer enhances the immobilization efficiency to a large extent. ${ }^{3}$ These ssDNA probes are then hybridized with the complementary ssDNA from the sample for the detection of specific pathogens. The PNA, a mimic of DNA with pseudopeptide backbone, has opened a new research area in the field of genosensors including detection of SNPs. The PNAs have a very high affinity for the DNA to form PNA-DNA hybrid.

\section{Microfluidic sensors}

Microfluidic devices with micron scale fluid channels and sensing chambers provide an efficient platform to develop genosensors. These devices are generally simple, sensitive, and robust. Microfluidics genosensor involves aggregation of bacterial cells through icrofabricated structures, magnetic beads or dielectrophoresis, lysis of the bacterial cells by thermal, chemical or mechanical forces, extraction of DNA, purification, and detection. ${ }^{22}$ Some of the DNAs also require PCR amplification. Kim et al., ${ }^{23}$ developed an electrochemical microfluidics sensor for the detection of $E$. coli from water samples. Wojcik et al., ${ }^{24}$ fabricated a microfluidic based DNA sensor for detection of M. tuberculosis. Wang et al. ${ }^{25}$ reported a microfludics sensor consisting ssDNA-Cy3/Au. These ssDNA$\mathrm{Cy} 3 / \mathrm{Au}$ exhibited fluorescence enhancement after hybridizing with complementary targets with a LOD of $1 \mathrm{pM}$.

\section{Sensor array}

A sensor array is a group of sensors arranged in a certain pattern to increase the signal-to-noise ratio: magnify the signal while suppressing the noise. The sensor arrays can detect the direction and distance of impinging signal sources. ${ }^{26}$ The technology to achieve this is called array signal processing which includes wireless communications, seismology, and fault diagnosis. A nanogap sensor for detection of DNA was reported by Roy et al. ${ }^{27}$ based on the electronic transduction mechanism by using standard silicon microfabrication technologies. The LOD of the sensor was obtained to about $1.0 \mathrm{fM}$ DNA. Canete \& $\mathrm{Lai}^{28}$ reported a 3-pixel electrochemical DNA sensor array with each pixel functionalized with a different stem-loop DNA probe utilizing potential-assisted "click" chemistry. They optimized the parameters of potential-assisted "click" chemistry using surrogate probe and then used them to fabricate the sensor array.

\section{Conclusion}

We have covered the recent progress in the genosensors for detection of pathogenic bacteria from food, environment or living organisms. The uses of NPs increase the sensitivity of the sensor to a large extent. However, there still remains a challenge to develop a practically applicable and portable device. The reliability, repeatability, durability, and ease-of operation remains an important issue for lab-on-a-chip pathogen sensors. In addition to this, integration of a complex process within a single chip still remains a challenge. A common issue in microfluidic devices is a clogging problem caused by non-selective binding that significantly reduces the reliability and life time of the sensor. 


\section{Acknowledgements}

The authors thank to Mrs. Sanjukta Satapathy, Principal Chikiti College, Chikiti and Dr. Sanjay Kumar, Director AcSIR-IGIB, for their valuable suggestions and corporations.

\section{Conflict of interest}

The author declares no conflict of interest.

\section{References}

1. Kumar A, Dash SK. DNA based biosensors for detection of pathogens. In: Singh HP, Chowdappa P, editors. Molecular approaches for plant fungal disease management. India: Westville Publishers; 2012. p. 31-35.

2. Khan M, Khan AR, Shin JH, et al. A Liquid-crystal-based DNA biosensor for pathogen detection. Scientific Reports. 2016;6:22676.

3. Dash SK, Sharma M, Kumar A, et al. Carbon composite-based DNA sensor for detection of bacterial meningitis caused by $N$. meningitidis. $J$ Solid State Electrochem. 2014;18(10):2647-2659.

4. Poltronieri P, Mezolla V, Primiceri E, et al. Biosensors for the detection of food pathogens. Foods. 2014;3(3):511-426.

5. Ligaj M, Tichoniuk M, Gwiazdowska D, et al. Electrochemical DNA biosensor for the detection of pathogenic bacteria Aeromonas hydrophila. Electrochimica Acta. 2014;128:67-74.

6. Ahmed A, Rushworth JV, Hirst NA, et al. Biosensors for whole-cell bacterial detection. Clin Microbiol Rev. 2014;27(3):631-646.

7. Rella R, Spadavecchia J, Manera MG, et al. Surface plasmon resonance imaging technique for nucleic acid detection. Biosens Bioelectron. 2004;20:1140-1148.

8. Hsu SH, Lin YY, Lu SH, et al. Mycobacterium tuberculosis DNA detection using surface plasmon resonance modulated by telecommunication wavelength. Sensors. 2014;14(1):458-467.

9. Cheng XR, Hau BY, Endo T, et al. Au nanoparticle-modified DNA sensor based on simultaneous electrochemical impedance spectroscopy and localized surface plasmon resonance. Biosens Bioelectron. 2014;53:513518.

10. Weihong JL, Wang K, Xiao D, et al. Ultrasensitive optical DNA biosensor based on surface immobilization of molecular beacon by a bridge structure. Anal Sci. 2001;17(10):1149-1153.

11. Cornett EM, Kolpashchikov DM. SNP Analysis using a molecular beaconbased operating cooperatively (OC) sensor. Mol Biol. 2013;1039:81-86.

12. Jiang D, Liu F, Liu C, et al. An electrochemical sensor based on allosteric molecular beacons for DNA detection of Escherichia coli. O157:H7. Int J Electrochem Sci. 2013;8(2013):9390-9398.

13. Cai Z, Song Y, Wu Y, et al. An electrochemical sensor based on label-free functional allosteric molecular beacons for detection target DNA/miRNA. Biosens Bioelectron. 2013;41:783-788.
14. Abu-Salah KM, Zourob MM, Mouffouk F, et al. DNA-based nanobiosensors as an emerging platform for detection of diseases. Sensors. 2015;15(6):14539-14568.

15. Inbaraj BS, Chen BH. Nanomaterial-based sensors for detection of foodborne bacterial pathogens and toxins as well as pork adulteration in meat products. J Food Drug Aanalysis. 2016;24:15-28.

16. Zhang CY, Yeh HC, Kuroki MT, et al. Single-quantum-dot-based DNA nanosensor. Nat Mater. 2005;4:826-831.

17. Rijal K, Mutharasan R. A Method for DNA-based detection of $E$. coli $\mathrm{O} 157: \mathrm{H} 7$ in a proteinous background using piezoelectric-excited cantilever sensors. Analyst. 2013;138(10):2943-2950.

18. Li C, Yang Y, Zhang B, et al. Conjugation of graphene oxide with DNAmodified gold nanoparticles to develop a novel colorimetric sensing platform. Part Part Syst Charact. 2013;31(2):201-208.

19. Dash SK, Sharma M. Diagnostic techniques and treatment of meningitis. In: Houllis G, Karachalios M, editors. Meningitis: causes, diagnosis and treatment. USA: Nova Science Publishers Inc; 2012. p. 203-223.

20. Patnaik S, Dash SK, Sethi D, et al. Engineered polymer-supported synthesis of 3'-carboxyalky modified oligonucleotides and their applications in the construction of biochips for diagnosis of the diseases. Bioconjugate Chem. 2012;23(3):664-670.

21. Dash SK, Sharma M, Khare S, et al. rmpMGenosensor for detection of human brain bacterial meningitis in cerebrospinal fluid. Appl Biochem Biotechnol. 2013;171(1):198-208.

22. Yoon JY, Kim B. Lab-on-a-chip pathogen sensors for food safety. Sensors. 2012;12(8):10713-10741.

23. Kim U, Ravikumar A, Seubert J, et al. Detection of bacterial pathogens through microfluidic DNA sensors and mobile interface toward rapid, affordable, and point-of-care water monitoring. IEEE J Transl Eng Health Med. 2013;1:3700207.

24. Wojcik BI, Lopes P, Catarina VA, et al. Bio-microfluidic platform for gold nanoprobe based DNA detection-application to Mycobacterium tuberculosis. Biosens Bioelectrn. 2013;48:87-93.

25. Wang J, Aki M, Onoshima D, et al. Microfluidic biosensor for the detection of DNA by fluorescence enhancement and the following streptavidin detection by fluorescence quenching. Biosens Bioelectron. 2014;51:280285.

26. Lonsdale CL, Taba B, Queralto N, et al. The use of colorimetric sensor arrays to discriminate between pathogenic bacteria. PLOS One. 2013;8(5):e6272.

27. Roy S, Chen X, Li MH, et al. Mass-produced nanogap sensor arrays for ultrasensitive detection of DNA. JACS. 2009;131(34):12211-12217.

28. Canete SJP, Lai RY. Fabrication of an electrochemical DNA sensor array via potential-assisted "Click" Chemistry. Chem Commun. 2010;46:39413943. 